\title{
Kunst als Wunde
}

\section{Vulnerabilität in der Bildenden Kunst seit ihrem Aufkommen in der Neuzeit und bei Joseph Beuys}

Marco A. Sorace

Um zu verstehen, welche Bedeutung das Motiv der Wunde für die Kunst hat, müssen wir uns vorab zweierlei klar machen: Zum einen, was wir hier als "Bildende Kunst« in einem engeren Sinne verstehen wollen und zum anderen - diese beiden Aspekte hängen freilich eng zusammen -, in welcher Weise diese Kunst sichtbar in Erscheinung tritt. Bei ersterem geht es also um gewisse Definitionsfragen und bei letzterem um die Frage nach einer Art "Phänomenologie der Kunst«. Sollen die Antworten, die wir diesbezüglich geben, fundiert sein, müssen wir noch einen weiteren Schritt zurückgehen und klären, aus welchem Anspruch an Bilder ein Verständnis des »künstlerischen" Bildes überhaupt erst erwachsen ist.

Rufen wir uns dafür zunächst eine grundlegende Unterscheidung der Bildtheologie in Erinnerung. In der jüdisch-christlichen Tradition des ersten Jahrtausends, die wir hier etwas genauer als Tradition eines jüdisch-christlichen Platonismus bezeichnen wollen, gibt es jene Kritik, wonach Gott oder das (absolute) Sein nicht auf ein Geschöpfliches oder ein Seiendes reduziert werden sollte. Alles andere wäre, insbesondere wenn dieses Seiende einen bewusst abbildhaften Bezug zum göttlichen Urbild hat, Idolatrie - also das Anhaften der Wahrnehmung an Idolen, kurzum "Gottesverkleinerungen«. Dagegen wurde in der christlichen Tradition seit der zweiten Hälfte des ersten Jahrtausends ein Verständnis von Bildern als Ikonen etabliert. Eine ikonische Wahrnehmung setzt, vereinfacht gesagt, voraus, dass die Ikonen betrachtet werden als Repräsentanten göttlicher Wahrheit, in der Weise, dass sie auf jenes Größere - das göttliche Urbild - abbildend verweisen. ${ }^{1}$ Die Ikonen dürfen aber nicht - so die traditionelle Bildtheologie seit dem byzantinischen Bilderstreit und dem II. Konzil von Nicäa - angebetet werden in dem Sinne, dass man an sie glaubt wie man an den unendlichen Gott glaubt.

\footnotetext{
1 Mit Frage nach einer Phänomenologie der "idolischen" und »ikonischen« Wahrnehmung hat sich v.a. der französische Philosoph Jean-Luc Marion befasst. Eine der präzisesten und verständlichsten Darstellungen dieser Forschung findet sich in:Tobias Specker, Einen anderen Gott denken? Zum Verständnis der Alterität bei Jean-Luc Marion, Frankfurt a.M. 2002.
} 
Im Folgenden soll nun skizziert werden, wie die Wunde in der Ikonenmalerei vorkommt. Sodann werfen wir einen Blick auf das neuzeitliche Verständnis der Wunde in der Kunst der Neuzeit, um schließlich am Beispiel von Joseph Beuys nachzuvollziehen, wie dieses Motiv zum Inbegriff und Sinnbild der Kunsterfahrung selbst werden konnte.

\section{Die Wunde in der Ikonenmalerei}

Interessant ist es, den Blick bereits hier auf einen bestimmten Ikonentypus und ein Detail desselben zu richten, nämlich auf den Typus der Kreuzigung und die Seitenwunde Jesu Christi. Die traditionelle Ikonographie sah im Lanzenstich des "Longinus ${ }^{2}$ und in dem aus der Wunde ausfließenden Blut den Ursprung des eucharistischen Sakraments und folglich auch den Ursprung der Kirche (Abb. 1). Man könnte also sagen:Aus der Wunde Christi fließt sein göttliches Leben in die kirchliche Kommunion. Die Ikone jedoch - in der liturgischen Praxis ist sie als Kultbild selbst Bestandteil dieses Kults ${ }^{3}$ - bildet nur ab, was sich letztlich auf einer anderen Ebene vollzieht. So ist das Bild als Ikone im strengen Sinne "Medium» der Offenbarung und vermittelt zwischen Gott und dem Betrachter.

Die damit markierte Begrenzung der Offenbarungsmöglichkeiten des Bildes war in den bildtheologischen Auseinandersetzungen des ersten Jahrtausends in der Tat heftig umstritten. Jenseits der Kontroverse darüber, ob eine Ikone Gott vergegenwärtigen kann, herrschte allerdings Einigkeit darüber, dass es einen wesentlichen Unterschied zwischen Urbild und Abbild gibt. ${ }^{4}$ In jedem Fall wird man also sagen müssen, dass eine wirklich religiöse Erfahrung ausschließlich in der Auseinandersetzung mit einem Bild - etwa der Kreuzigungsikone und der Darstellung der Wunden des Gekreuzigten - im Rahmen der alten Ikonentheologie sicher undenkbar war.

\footnotetext{
2 Der in der Ikonographie einen festen Platz einnehmende römische Hauptmann "Longinus", welcher nach der apokryphen Erzählung des Nikodemusevangeliums die Seite Christi mit dem Speer geöffnet hat, wird somit auch positiv bewertet und galt sogar als einer der ersten Zeugen des christlichen Glaubens.

3 Vgl. dazu auch: Hans Belting, Bild und Kult. Eine Geschichte des Bildes vor dem Zeitalter der Kunst, München ${ }^{5} 2000,175-184$.

4 Die dogmatische Unterscheidung auf dem II. Konzil von Nicäa (787) zwischen der "Prokynesis" (der "Verehrung", die den Bildern zukommen soll) und der "Latreia" (der "Anbetung", die allein Gott zukommen kann) unterstreicht gewissermaßen diese beidseitige Akzeptanz. Sehr gut dargestellt sind die theologischen Diskussionen, die zu dieser Entscheidung geführt haben, in: Christoph Schönborn, Die Christus-Ikone. Eine theologische Hinführung, Schaffhausen 1984.
} 


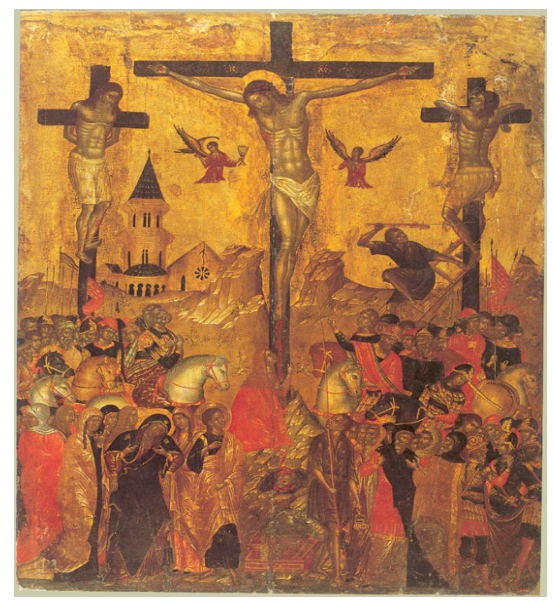

Abb. 1: Kreuzigungsikone aus Kreta, 17. Jahrhundert (zahlreiche Traditionen des ersten Jahrtausends aufnehmend), Eremitage, St. Petersburg

\section{Neuzeitliche Kunst: Das Bild als Wunde}

Auf der Schwelle zur Neuzeit kommt es zu einem allmählichen Ablösungsprozess des Bildes aus seiner kultischen und institutionellkirchlichen Verbindung. Das neuzeitliche Bild führt zwar zunächst anders ist es in Bezug auf seinen geistesgeschichtlichen Ursprung gar nicht denkbar - in bestimmter Weise den spätantiken und mittelalterlichen Begriff des Bildes als Medium der Wahrheit fort, jedoch wird der Künstler nun mehr und mehr zu einem Akteur, der in das Geschehen zwischen Betrachter und Bild eingreift. Er agiert als einer, der in seinem Schaffensprozess auf unterschiedliche Weise die Oberfläche, die Haut des Bildes, aufreißt und so gleich dem Apostel Thomas in die Wunde der göttlichen Offenbarung greift (Abb. 2). Für die künstlerische Wahrnehmung wird wie auf dem Bild von Caravaggio die Berührung bzw. die leiblich-affektive (Selbst-)Erprobung bedeutsam. Oder einfacher ausgedrückt: In der sinnlichen Wahrnehmung selbst - im Tasten, Sehen usw. - offenbart sich die Gabe des Lebens. Hervorzuheben ist dabei, dass hier das Verständnis einer "autonomen Kunst« aufkommt, in deren Zuge die Bildandacht und die hier erst aufkommende "Bildende Kunst« im engeren Sinne zu einem hinreichenden Erfahrungsraum des Religiösen wird. 


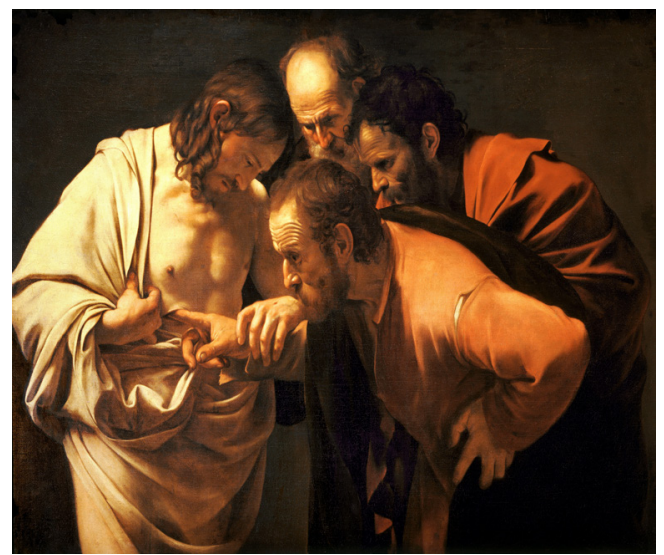

Abb. 2: Caravaggio, Der Unglaube des heiligen Thomas, um 1601, Öl auf Leinwand, Schloss Sanssouci, Potsdam

Es ist nun aber auch die eingangs gestellte Frage nach der »Phänomenologie der Kunst" anzusprechen. In den letzten beiden Jahrzehnten hat es diesbezüglich in der Kunstkritik und Ästhetik eine auch religionshermeneutisch nicht unbedeutsame Diskussion um die Möglichkeit und Unmöglichkeit der "Berührung" gegeben: Jacques Derrida und Jean-Luc Nancy führten diesen Diskurs an und beriefen sich dabei zuerst auf Aristoteles. Aristoteles habe in seiner Schrift »Über die Seele« die Möglichkeit einer gelingenden Berührung ausgeschlossen, da beim Versuch des Berührens zwischen Berührenden und Berührten nichts Vermittelndes auszumachen sei (vgl. Peri psychēs 422b). Derrida sieht hinter dem Wunsch, das Andere oder den Anderen zu berühren, eine ontologische Sehnsucht nach dem Seinsursprung am Werk, jedoch zeige sich im stetigen Scheitern der Sehnsuchtserfüllung durch Berührungsversuche jenes "unbezwingbare Prinzip«, demnach jede Sinngebung einen Aufschub von Sinn, eine uneinholbare Differenz bedeute. Nancy meint dabei, die besondere Affinität der Maler und Künstler zu Bildthemen der Berührung wie etwa das "Noli me tangere" begründe sich darin, dass sie um das Prinzip der Unberührbarkeit im Offenbarungsgeschehen wüssten. ${ }^{5}$

\footnotetext{
5 Vgl. Jacques Derrida, Berühren. Jean-Luc Nancy, Berlin/Zürich 2007 (frz.: Le toucher. Jean-Luc Nancy, Paris 2000), 137; vgl. dazu auch Jean-Luc Nancy, Noli me
} 
Dagegen haben Michel Henry und in Anschluss an diesen Rolf Kühn versucht darzulegen, wie die Berührung dennoch phänomenologisch aufweisbar ist. Für Henry gründet die Möglichkeit der Berührung in einer ursprünglichen "Selbstberührung«, einem inneren Berührtsein als einem Sich-selbst-offenbar-Werden im Leben. "Was wäre«, so fragt Kühn im Blick auf den Zusammenhang von Berührung und Kunst, "nämlich ein künstlerisches Werk ohne innere >Betroffenheit des Rezipierenden? ${ }^{6}$

Tatsächlich lassen die in der Kunst immer wieder und auf vielfache Weise zu beobachtenden Versuche, die Oberfläche des Bildmediums aufzubrechen oder -zu reißen - wie etwa hier ganz sinnfällig bei Lucio Fontana (Abb. 3) - kaum vereinbaren mit der Theorie einer grundsätzlichen Unberührbarkeit und der absoluten Reduktion auf immer neue Oberflächen, wie sie von Derrida und Nancy vertreten wird. Und auch eine so extreme Kunstpraxis wie die von Gina Pane, die in Aktionen der 1970er Jahre mit einer Rasierklinge "Bildzeichen « in die eigene Haut ritzte, lässt sich nicht erklären, ohne die Tatsache, dass solche Inszenierungen einerVerwundung die Betrachter gewissermaßen berühren, indem die Erfahrung der Wunde sie zunächst einmal selbst berührt. Dies ist die Voraussetzung mit der wir uns nun diesem Thema bei Joseph Beuys zuwenden können.

\footnotetext{
tangere, Berlin 2008 (frz.: Noli me tangere. Essai sur la levée du corps, Paris 2003), hier bes. $66 f$.

6 Vgl. Michel Henry, Inkarnation. Eine Philosophie des Fleisches, Freiburg/München 2002 (frz.: Incarnation. Une philosophie de la chair, Paris 2000), hier 216ff.; vgl. dazu auch Rolf Kühn, Praxis der Phänomenologie. Einübungen ins Unvordenkliche, Freiburg/München 2009, 297-320, hier 298; vgl. dazu auch Marco A. Sorace, Das Minimum als Maximum. Rolf Kühns "Elementarästhetik « im Kontext gegenwärtiger Kunsttheorie, in: Sophia Kattelmann/Sebastian Knöpker (Hg.), Lebensphänomenologie in Deutschland. Hommage an Rolf Kühn, Freiburg/München 2012, 168-181.

7 Vgl. ausführlicher zur Aktionskunst von Gina Pane: Karoline Künkler, Aus den Dunkelkammern der Moderne. Destruktivität und Geschlecht in der Bildenden Kunst des 19. und 20. Jahrhunderts, Köln 2012, $583 f f$.
} 


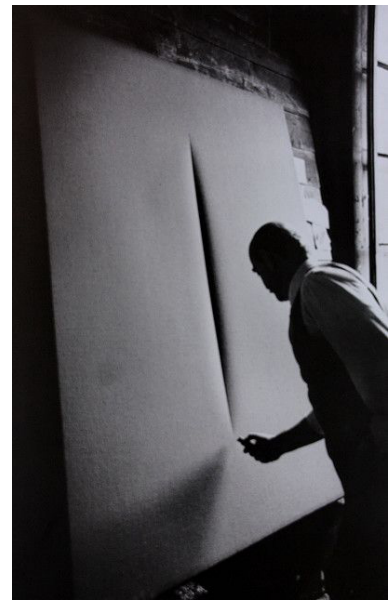

Abb. 3: Das Bild zeigt den Künstler Lucio Fontana während der Arbeit (»Tagli« "Schnitte") an einer Leinwand aus seiner Werkphase "Concetto spaziale - Attese" ("Raumkonzept - Erwartungen«), (C) Pro Litteris / Photo Ugo Mulas (C) Ugo Mulas Heirs. All rights reserved.

\section{Joseph Beuys: »Ausstellung« der Wunde}

Man kann mit Recht behaupten, dass die Wunde für den Kunstbegriff von Joseph Beuys eines der zentralsten Motive darstellt. Steht es doch gewissermaßen am Anfang seines großen Auftretens in den frühen 1960er Jahren unter anderem in Aachen im Rahmen einer aufsehenerregenden Kunst-Aktion (zum »Festival der neuen Kunst«, 1964 im Audimax der TH), anlässlich derer der Künstler ein Typoskript mit dem Titel "Lebenslauf-Werklauf» verfasst hat. Dieses eröffnete er mit einem Eintrag: »1921 - Kleve - Ausstellung einer mit Heftpflaster zusammengezogenen Wunde«. ${ }^{8}$ Und auch am Ende seines Wirkens in seinem vorletzten öffentlichen Vortrag über Sprache, soziale Plastik und Auferstehung findet sich wiederum dieses

\footnotetext{
8 Ein erster, mit handschriftlichen Anmerkungen versehener Entwurf dazu befindet sich im Joseph-Beuys-Archiv in Schloss Moyland. Eine Transkription des Blattes findet sich u.a. in: Götz Adriani/Winfried Konnertz/Karin Thomas, Joseph Beuys. Leben und Werk 1986, Köln ${ }^{3} 1986,11-13$.
} 
Motiv, wenn er diesen einleitet mit den Worten "Es ist auch jetzt wieder so, dass ich anfangen möchte mit der Wunde. «"

Joseph Beuys selbst ist in seinem Leben vielfach verwundet worden - konkret-körperlich als Soldat im Zweiten Weltkrieg, aber auch im übertragenen Sinne in den Jahren danach, nachdem er als Künstler versuchte, über seine Verwundbarkeit hinwegzuarbeiten. Eine künstlerische Karriere schien in dieser Zeit weit entfernt. Am Ende einer tiefen Lebenskrise um das Jahr $1957^{10}$ steht dann seine Einsicht: Das, was aus den Lebenswunden austritt, muss schöpferisch verwandelt werden. Das eigenständig-schöpferische Leben beginnt gewissermaßen mit der Wunde der Abnabelung - 1921 ist ja Beuys' Geburtsjahr. So sieht er in der Abnabelungswunde den ursprünglich künstlerischen Auftrag an jeden Menschen, die durch die trennende Wunde verlorene Gemeinschaft wieder herzustellen. Diesen Auftrag buchstabiert er in Objekten, Installationen und Aktionen durch, wie beispielsweise die mit Wundmaterialien versehene »Kinderbadewanne" von 1960, die Rauminstallation "Schmerzraum"von 1983 oder auch schon die frühe, auf die Verwundung und Krise des Ignatius von Loyola bezugnehmende Aktion »Manresa» von 1966 - jeweils nur relativ willkürlich ausgewählte Beispiele. Man könnte diese Liste umfangreich fortfuihren. Allen diesen Arbeiten gemeinsam ist, dass sie letztlich auf den anthropologisch erweiterten Kunstbegriff bzw. auf die soziale Plastik als Beuys' eigentliches Kunstwerk hinweisen, also auf ein schöpferisches Potenzial das allen Menschen gemeinsam ist. ${ }^{11}$

9 Erstmals veröffentlicht im Jahr 1985 unter dem Titel »Reden über das eigene Land: Deutschland 3«, hier zit. nach: Joseph Beuys, Das Geheimnis der Knospe zarter Hülle. Texte von 1941-1986, hg. v. Eva Beuys, München 2000, 24-39 (Manuskript zu »Sprechen über das eigene Land: Deutschland«.Vortrag, gehalten am 20. November 1985, 3. Münchner Kammerspiele), 25.

${ }^{10}$ Es hat in den letzten Jahren eine Reihe genauso wenig respektvoller wie sachorientierter Kritiken an Beuys' Bewältigung seiner und der deutschen Vergangenheit gegeben - zuletzt und diese weitgehend zusammenfassend in einem umfangreichen Buch von Hans Peter Riegel (ders., Beuys. Die Biographie, Berlin 2013). Was dazu unbedingt zu sagen ist, findet sich u.a. in: Marco A. Sorace, Beuys - Eine Biographie. Eine kritische Auseinandersetzung (www.theomag.de/85/mas1.htm).

${ }^{11}$ Im Gespräch mit dem Jesuiten Friedhelm Mennekes stellte Beuys fest, was sein wichtigster Beitrag zum "Christusbild" in der Kunst des 20. Jahrhunderts sei. Auf die Nachfrage von Mennekes nach einem konkreten Werk, das diesen repräsentiere, erwiderte Beuys: „Der erweiterte Kunstbegriff. Ganz einfach." Das sei ein wirkliches Bild, aber keines, »das irgendwo an einer Wand hängt«. Dagegen weisen diese »äußeren Bilder", so Beuys, bei ihm lediglich auf dieses zentrale "Werk", den erweiterten Kunstbegriff, hin.Vgl. dazu Friedhelm Mennekes, Beuys zu Christus. Eine Position im Gespräch / Beuys on Christ. A Position in Dialogue, Stuttgart 1990, 60-63. 
Wenn es um die Wunde geht, gibt es natürlich dennoch ein besonderes Werk, das fast jedem in den Sinn kommt, der ein wenig mit Beuys vertraut ist: Gemeint ist die zwischen 1974 und 1975 entstandene Münchner Rauminstallation "Zeige deine Wunde» (Abb. 4). ${ }^{12}$ Bei diesem Objektensemble fallen zuerst zwei in der hinteren linken Ecke des Raums stehende Bahren ins Auge, wie sie in der Pathologie verwendet werden und die ein Abflussloch für das aus den letzten Wunden austretende Blut haben. Unmittelbar unter diesen Abläufen befinden sich jeweils Zinkblechkästchen, die mit Fett gefüllt und je einem Fieberthermometer sowie einem Reagenzglas versehen sind. Jedes der beiden Reagenzgläser beinhaltet am Ende ein Schädelchen eines Singvogels. Das vergossene und aufgefangene Blut könnte hier in einem bildlichen Sinne in einen neuen Energie- und Wärmeprozess eintreten und so in einem weiteren Schritt das, was aus dem Vogelkopf hervorkommen kann - der schöne Gesang als ein Symbol für das Künstlerische überhaupt -, zu einem neuen Leben erwecken. Die übrigen darauf bezogenen Objekte im Raum, etwa die »Schepser" (Werkzeuge, mit denen man Bäume von der Rinde befreit, um einen fatalen Schädlingsbefall des Waldes zu bekämpfen) oder die gerahmten Ausgaben der italienischen kommunistischen Zeitung »La Lotta Continua«, sind genauso wie die Bahren allesamt als Doppelobjekte angelegt, was bei Beuys stets ein Hinweis auf ein Tun in der Gemeinschaft ist. Weitere Botschaften - weil die Wunde auf die die Menschen verbindende schöpferische Lebendigkeit verweist - wären entsprechend: Das Zeigen der Wunde hat - mit Blick auf die beiden Schultafeln - die Dimension eines gemeinsamen Lernprozesses, es hat eine Dimension einer sozial gestalteten Politik, eines geteilten Kapitals usw.

In dieser und in anderen Arbeiten von Joseph Beuys geht es also zentral um Vulnerabilität, es geht darum, Verwundbarkeit zu zeigen, damit die Wunde heilen kann. Aber nicht nur dies, sondern auch jenes, was für den Mensch als Künstler aus diesem Prozess resultiert: Die gezeigte, ausgesetzte oder ausgestellte Wunde ruft nach einer Wandlung, einem Transformationsprozess - wir sprechen in diesem Zusammenhang heute oft von Resilienz. Diese "Wandlung" kann und sollte man durchaus im Sinne von Beuys ${ }^{13}$ auch kultisch

${ }^{12} \mathrm{Zu}$ dieser Installation und insbesondere auch zu den teilweise erschütternden öffentlichen (an die "Entartete-Kunst-Diktion" anknüpfenden) Reaktionen zur Ausstellung dieser Arbeit in einer Unterführung auf der Münchner Maximilianstraße im Jahre 1976 vgl. Armin Zweite, Zeige deine Wunde, München 1976.

${ }^{13}$ Beuys hatte dabei durchaus eine kultisch-religiöse Transformation im Sinn, aber entschieden nicht im Rahmen des kirchlich-institutionellen Christentums. Am deutlichsten spricht über diese Abgrenzung mit dem evangelischen Theologen Horst 
verstehen, jedoch jenseits von spitzfindig kirchlich-theologischen Auseinandersetzungen, die sich manifestiert haben etwa in begrifflichen Abgrenzungen wie Transsubstantiation, Konsubstantiation oder Transsignifikation. ${ }^{14}$ Was diese "Wandlung" dagegen bedeuten könnte, wird vielleicht noch am deutlichsten an Beuys'Verständnis des Leidens, das für ihn eine wirklichkeitsverändernde Kraft, ja sogar "die« wahrhaft verändernde Kraft ist: »Denn das Leiden ist natürlich ein bestimmter Ton in der Welt. Er ist hörbar. Man sieht ihn wohl auch. Wer sich einmal anstrengt, solches wahrzunehmen, der sieht im Leiden eine ständige Quelle der Erneuerung. Es ist eine Quelle von kostbarer Substanz, die das Leiden in die Welt entlässt. Da sieht man: eine unsichtbar-sichtbare sakramentale Substanz. ${ }^{15}$

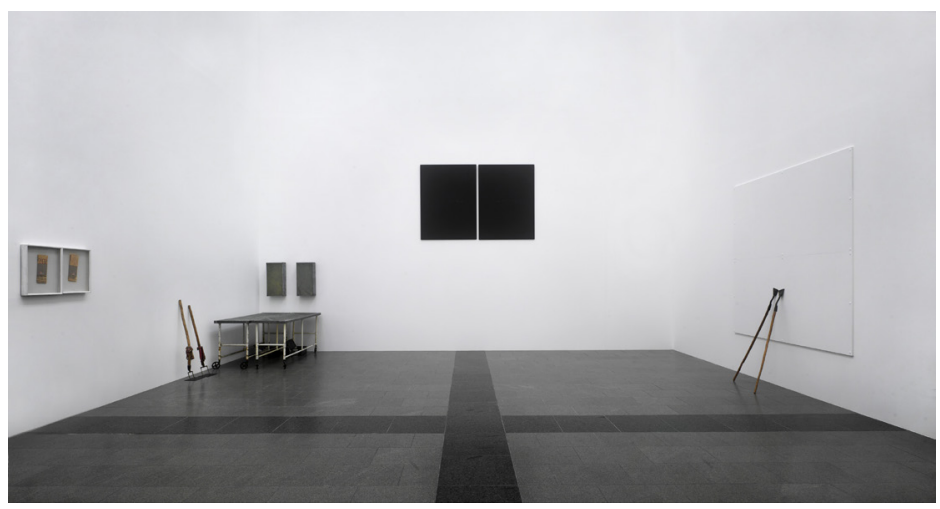

Abb. 4: Joseph Beuys, Zeige deine Wunde, 1974-1975, Rauminstallation, Städtische Galerie Lenbachhaus, München, (C) Pro Litteris

Schwebel, in: ders., Glaubwürdig - Fünf Gespräche über heutige Kunst und Religion. München 1979, 15-42, hier 28ff.

${ }^{14}$ Vgl. zum Thema der Wandlung im Werk von Joseph Beuys die Ausführungen des österreichischen Priesters und Avantgarde-Galeristen Otto Mauer, in: ders., Über Kunst und Künstler, hg. v. Günter Rombold, Salzburg 1993, 260-269 (»Rede über Joseph Beuys").

${ }^{15}$ Mennekes, Beuys zu Christus, 46. 
Am Beispiel von Joseph Beuys sollte angezeigt werden, wie die Wunde in der Kunst keineswegs ein Motiv in einem bloß bildgegenständlichen Sinne geblieben ist.Vielmehr sind sich Künstler bewusst, dass jene Transformationsprozesse, die mit der Wunde verbunden sind, sich als Inbegriff und Sinnbild des kreativ-künstlerischen Prozesses anbieten und zwar so, dass man sich fragen kann, ob die Erfahrung der Wunde nicht sogar noch über diese sinnbildliche Dimension hinaus der eigentliche Beweggrund der künstlerischen Praxis ist. Beuys jedenfalls hätte diese Frage bejaht.

- Dr. Marco A. Sorace ist Dozent an der Bischöflichen Akademie im Bistum Aachen. Zu seinen Arbeitsschwerpunkten gehören insbesondere die Bildtheologie, der Dialog von Kunst und Kirche sowie die Theologie der Spiritualität und Mystik. 